\title{
Synthesis and Characterization of Styrene, Maleic Anhydride and N-phenyl Maleimide Tripolymer
}

\author{
Y. L. MOU, W. XIA, Z. WANG, D. F. ZHENG* \\ School of Biochemical Chemical Engineering, Hainan Institute of Science and Technology, Haikou, \\ 571100, PR China \\ *Corresponding author: 407484467@qq.com
}

Keywords: Terpolymer; characterization; thermal resistance; DSC/TG test

\begin{abstract}
A terpolymer resin (NSMA) was synthesized with styrene, maleic anhydride and $\mathrm{N}$-phenyl maleimide. The structure and composition was characterized with FT-IR and ${ }^{13} \mathrm{C}-\mathrm{NMR}$. The thermal resistance of NSMA was determined through integrated thermal analysis. The results indicated that, when the molar ratio of N-PMI, St and MA was 1:3:1, the $T_{g}, T_{w t 10 \%}, T_{w t 20 \%}, T_{w t 50 \%}$, and $T_{\max }$ of NSMA was $227^{\circ} \mathrm{C}, 394^{\circ} \mathrm{C}, 409^{\circ} \mathrm{C}, 431^{\circ} \mathrm{C}$ and $434^{\circ} \mathrm{C}$, respectively, much higher than those of styrene/maleic anhydride copolymer. Furthermore, the thermal resistance of NSMA increased with the content of N-phenyl maleimide. The results showed that NSMA had a favorable application prospect in the thermal resistance resin.
\end{abstract}

\section{Introduction}

With the excellent mechanical properties, dielectric properties, thermal stability and outstanding bonding ability, epoxy resin has been widely applied as coating, insulation materials, etc, in the electronic, electrical, mechanical manufacturing, aerospace and other fields, especially in the E-insulating material. However, with the development of printed circuit board (PCB), the demand on the thermal resistance of insulation material becomes severe ${ }^{[1,2]}$, which the traditional epoxy resin could not meet. In recent years, scientists have modified the epoxy resin system with polyphenylene oxide (PPO), and have greatly improved the heat-resistance of the system ${ }^{[3]}$. However, PPO is a thermoplastic resin, which needs thermosetting modification in the application. With the high cost and complex process in the modification, the application of PPO has been limited in the high-performance printed circuit boards ${ }^{[4]}$. Styrene/maleic anhydride copolymer and styrene/maleimide copolymer also have favorable thermal resistance property ${ }^{[5,6]}$, but still could not meet the demands of PCB. Therefore, it is significant to develop a new thermosetting resin which can be utilized in the high-performance PCB.

It is known that N-phenyl maleimide (N-PMI) has high thermal resistance ${ }^{[7]}$. The molecule formula is shown in Fig.1.

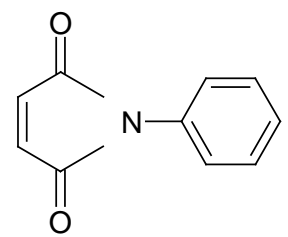

Figure 1 The molecular formula of N-PMI

There is a five-member ring in the N-PMI molecule. When it is embedded in a polymer chain, the internal rotation resistance of the chain would be increased, so does the thermal resistance of polymer ${ }^{[8]}$. Moreover, N-PMI has the properties of non-toxicity, favorable compatibility ${ }^{[9]}$. Thus, as a new heat-resistant co-monomer, N-PMI has attracted more and more attention.

In this study, N-PMI is introduced into the styrene and maleic anhydride copolymer system, to improve the thermal resistance of the system. The structure and composition of the product, a new $\mathrm{N}-\mathrm{PMI} /$ styrene/maleic anhydride polymer (NSMA), is characterized by nuclear magnetic resonance, 
infrared spectroscopy and elemental analysis. Further, the integrated thermal property is measured by DSC/TG. This work could explore the potential application of NSMA in the PCB.

\section{Experimental}

\section{Materials}

Maleic anhydride (MA), analytical grade, was obtained from Guangzhou Jinhuada Chemical Co., Ltd, China; styrene (St) and triethylamine, chemically pure, were obtained from Guangzhou Jinhuada Chemical Co., Ltd, China; benzoyl peroxide (BPO), analytical grade, was obtained from Guangzhou Reagent Factory, China; N-phenyl maleimide (N-PMI), chemically pure, were obtained from Guangzhou Reagent Factory, China.

\section{Synthesis of NSMA}

Solution polymerization method was adopted in this experiment. A mixed solvent of toluene/cyclohexanone (with a molar ratio of 1:1) was added into a 4-neck flask (fitted with a blender, funnel and thermomer), and then was heated to $90^{\circ} \mathrm{C}$. A certain amount of reactant monomers (consisted of MA, St and N-PMI) were dropped into the solvent slowly, together with $\mathrm{BPO}$ (as the initiator). The dropping process was finished in 4-5 h, and then the reaction system was kept for $2 \mathrm{~h}$ at $90^{\circ} \mathrm{C}$. After the reaction was terminated, the liquid was deposited with large number of methanol (or ethanol) under quick mixing, so as white floc was precipitated. The floc was re-crystallized at least three times, and then dried at $50^{\circ} \mathrm{C}$. The final result was NSMA. The copolymerization formula was as follows:

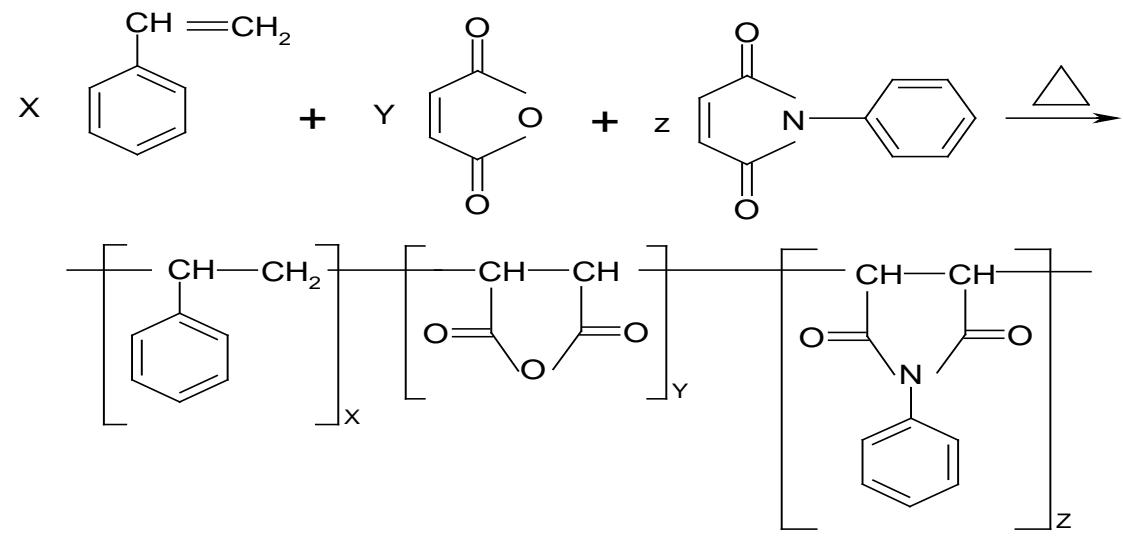

Figure 2 Scheme of copolymerization for NSMA

\section{Infrared spectroscopy}

The sample was compressed as a tablet with $\mathrm{KBr}$, the test was carried under a Nicolet 5700 fourier transform infrared spectrometer (from Thermo Nicolet CO., US).

\section{${ }^{13}$ C-NMR spectrum}

Refined polymer sample was dissolved with acetone first. The ${ }^{13} \mathrm{C}-\mathrm{NMR}$ test of the sample was conducted with a picoSpin nuclear magnetic resonance spectrometer (from Thermo Fisher Scientific CO., US).

\section{Integrated thermal analysis}

A TGA/DSC1 integrated thermal analyzer (from Mettler-Toledo CO., Switzerland) was adopted to measure the DSC/TG curves of the polymer and the initial thermal decomposition temperature. The experimental conditions are as follows, protection atmosphere: high-purity $\mathrm{N}_{2}$; ventilation rate: $20 \mathrm{~mL} / \mathrm{min}$; heating rate: $10^{\circ} \mathrm{C} / \mathrm{min}$; reference: $\alpha-\mathrm{Al}_{2} \mathrm{O}_{3}$. 


\section{Results and discussion}

\section{Infrared spectroscopy of NSMA}

In the infrared spectra of NSMA(synthesized at a molar ratio of 1:3:1(n(N-PMI):n(St):n(MA)), $1525 \mathrm{~cm}^{-1}$ and $1625 \mathrm{~cm}^{-1}$ is the vibration absorption of benzene skeleton; $1805 \mathrm{~cm}^{-1}$ and $1737 \mathrm{~cm}^{-1}$ is the absorption of $\mathrm{C}=\mathrm{O}$ in MA unit and N-PMI unit; $1210 \mathrm{~cm}-1$ and $1409 \mathrm{~cm}-1$ is the adsorption of the pentagon of MA unit and N-PMI unit ${ }^{[10]}$. In the spectra, there is no vibration absorption of $\mathrm{C}=\mathrm{C}$ bond. From the above, it can be deduced that N-PMI has been introduced into the polymer.

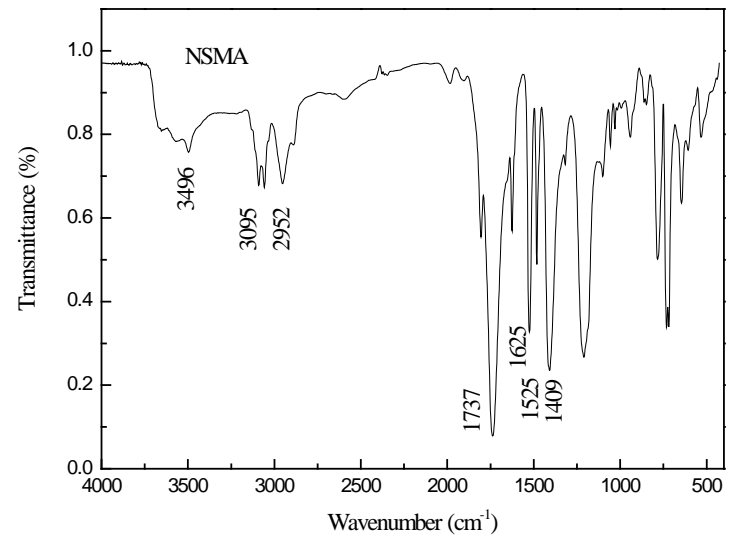

Figure 3 FT-IR spectrum of NSMA

\section{${ }^{13}$ C-NMR of NSMA}

In the ${ }^{13} \mathrm{C}$ NMR spectrum of NSMA, the chemical shift of $\delta=179.224$ is the characteristic absorption of carbonyl in MA; $\delta=129.596$ is the characteristic absorption of the quarternary carbon in the benzene ring, and $\delta=127.658$ is that of the tertiary carbon atom. From the spectrum, it can be further confirmed that the sample is the copolymer of styrene, MA and N-PMI.

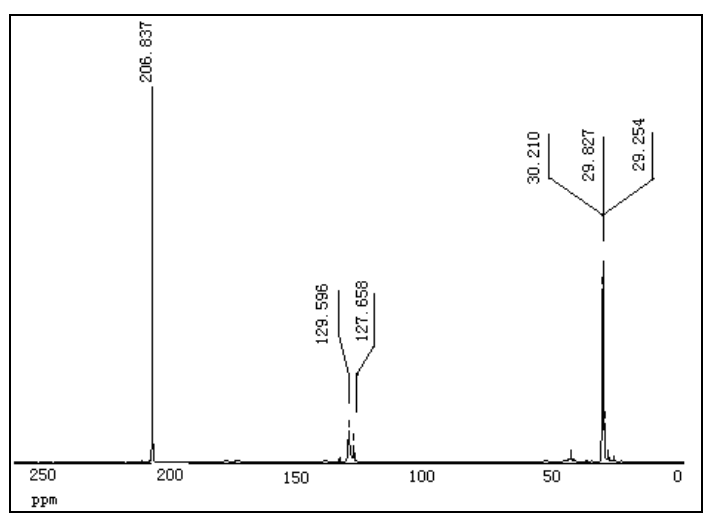

Figure $4{ }^{13} \mathrm{C}$ NMR spectrum of NSMA

\section{The thermal resistance of NSMA}

DSC test was conducted on NSMA; the results were shown as Fig.5. The control was SMA (synthesized at a molar ratio of 3:1 (n (St): $\mathrm{n}(\mathrm{MA})$ ).

From the DSC curve, the $T_{g}$ of NSMA is $227^{\circ} \mathrm{C}$, higher than that of SMA by $106^{\circ} \mathrm{C}$, indicating that the introduction of N-PMI increases the thermal resistance of the polymer obviously. The main reason is that the $\mathrm{N}$-PMI molecule contains a five-member ring, with a 1, 2-double substituted ethenyl in the structure, which has a higher steric resistance. When N-PMI is introduced into the molecular chain of NSMA, the rotation of the molecule is restricted, and the internal rotation resistance of the chain is increased, resulting in the reduction of the flexibility of the molecule, and the increasing of the stiffness ${ }^{[11]}$. Therefore, the thermal resistance of the polymer is increased. 
It can be also seen that, there is only one plat in the DSC curve of NSMA, presenting that this material has only one glass transition temperature, which further indicates that NSMA is a homogeneous copolymer synthesized with N-PMI, St and MA, rather than a mixture.

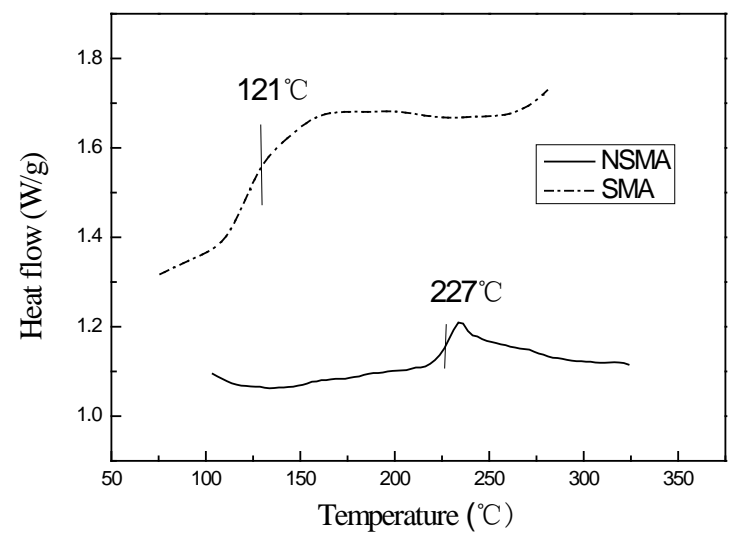

Figure 5 DSC test of NSMA

Fig.6 is the TG curve of NSMA and SMA. Temperature when material loses $10 \mathrm{wt} \%, 20 \mathrm{wt} \%$ and $50 \mathrm{wt} \%$ of its mass (marked as $T_{w t 10 \%}, T_{w t 20 \%}$ and $T_{w t 50 \%}$, respectively) and the temperature at the maximum weight-loss rate $\left(T_{\max }\right)$ are other important parameters indicating the thermal properties of materials. The results were listed in Table 1.

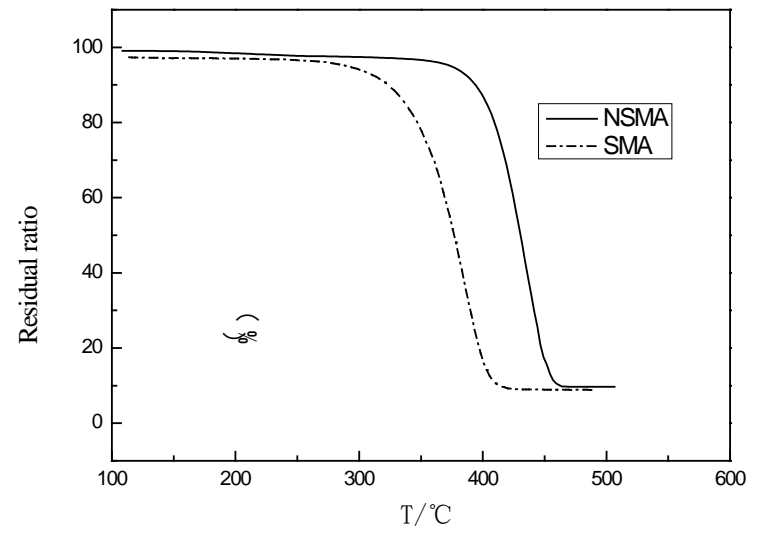

Figure 6 The residual ratio of NSMA as a function of temperature

From Table 1, the parameters of NSMA are all higher than those of SMA, especially for $T_{w t 10 \%}$, which is $71^{\circ} \mathrm{C}$ higher than that of SMA. When the material reaches the maximum weight-loss rate, the thermal decomposition temperature of NSMA is also $47^{\circ} \mathrm{C}$ higher than that of SMA. The reason is that the introduction of N-PMI increases the internal rotation resistance of the polymer molecule, resulting in the thermal decomposition curve of NSMA shifting to the direction of high temperature [12].

Table $1 T_{w t 10 \%}, T_{w t 20 \%}, T_{w t 50 \%}$ and $T_{\max }$ of NSMA

\begin{tabular}{ccccc}
\hline Sample & $T_{w t .10 \%} /{ }^{\circ} \mathrm{C}$ & $T_{w t .20 \%} /{ }^{\circ} \mathrm{C}$ & $T_{w t .50 \%} /{ }^{\circ} \mathrm{C}$ & $T_{\max } /{ }^{\circ} \mathrm{C}$ \\
\hline NSMA & 394 & 409 & 431 & 434 \\
SMA & 323 & 347 & 375 & 387 \\
\hline
\end{tabular}

\section{Effect of N-PMI content on the thermal resistance of NSMA}

Fig.7 showed the TG curves of NSMA with different N-PMI contents. From Fig.7, when the molar content of $\mathrm{N}-\mathrm{PMI}$ is $11.1 \%, 20.0 \%$ and $33.3 \%$, the temperature at the maximum decomposition rate is $370^{\circ} \mathrm{C}, 387^{\circ} \mathrm{C}$ and $420^{\circ} \mathrm{C}$, respectively. It also can be concluded that the 
thermal stability of NSMA improves with the increasing content of N-PMI.

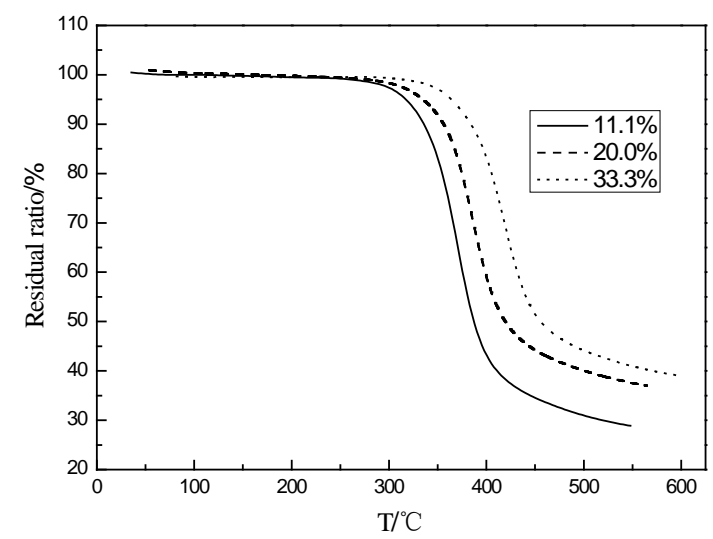

Figure 7 Effect of N-PMI content on the thermal resistance of NSMA

As for NSMA, the main factor of affecting the thermal properties is N-PMI, with a five-member ring in the molecular. The higher content of N-PMI, the larger the internal rotation resistance ${ }^{[13]}$, so the thermal stability of NSMA is higher.

\section{Conclusions}

1) A terpolymer(NSMA) was synthesized with solution polymerization method. FT-IR and ${ }^{13} \mathrm{C}-\mathrm{NMR}$ was applied to characterize the structure and composition. The results confirmed that NSMA is a homogeneous polymer.

2) The integrated thermal properties of NSMA were measured through DSC and TG test. The results indicated that when the molar ratio of N-PMI, St and MA was 1:3:1, the $T_{g}, T_{w t 10 \%}, T_{w t 20 \%}$, $T_{w t 50 \%}$, and $T_{\max }$ was $227^{\circ} \mathrm{C}, 394^{\circ} \mathrm{C}, 409^{\circ} \mathrm{C}, 431^{\circ} \mathrm{C}$ and $434^{\circ} \mathrm{C}$, respectively, much higher than those of SMA. Furthermore, the thermal stability of NSMA increases with the content of N-PMI. As a result, the introduction of N-PMI enhances the thermal resistance of the system obviously. Thus NSMA has a favorable application prospect in the thermal resistance resin.

\section{Acknowledgement}

This research was financially supported by Hainan Natural Science Fund (No.20155202).

\section{References}

[1] Lin, L.L., Ho, T.H. \& Wang, C.S., Synthesis of novel trifunctional epoxy resins and their modification with polydimethylsiloxane for electric application, Polymer, 38, pp.1997-2003, 1997.

[2] Watanabe, F., Mizoguchi, T. \& Iwafune, M., Polymer composition for electrical part material, US Patent 5,502,098, 1996.

[3] Zheng, M.S., Lin, Q.T. \& Kang, H.Z., Cross-linking polyphenyl ether resin with epoxy group, its composition and making method, CN Patent 1,385,454, 2002.

[4] Liang, G.Z. \& Gu, Y.J., Preparation method and application of modified double maleimide resin, CN Patent 1,493,600, 2004.

[5] Chernikova, E., Terpugova, P., Bui, C. \& Charleux, B., Effect of comonomer composition on the controlled free-radical copolymerization of styrene and maleic anhydride by RAFT, Polymer, 44, pp.4101-4107, 2003.

[6] Tawfik, M.E. \& Elsabee, M.Z., Preparation and characterization of polyester based on bis-(2-hydroxypropyl terephthalate, J Polym Res, 9, pp.129-133, 2002. 
[7] Thamizharasi, S. \& Reddy, B.S.R., Copolymerization of N-substituted maleimide with alkyl acrylate and its industrial applications, J Appl Polym Sci, 80, pp.1870-1879, 2001.

[8]Zhu, Z.Q., Shi, Z.J., Yuan, X.H. \& Du, G.Q., Study on the Thermal Properties of Copolymer SMA, China Synthetic Resin and Plastics, 16, pp.45-49, 1999.

[9] Teraki, S. \& Yoshida, M., Thermosetting film for die bonding and method of manufacturing semiconductor apparatus uses the same. JP Patent 2,009,111,276, 2009.

[10] Suzuki, K., Epoxy resin composition, JP Patent 8,134,328, 2006.

[11] Chang, J.Y., Kim, T.J., Han, M.J., Choi, D.H. \& Kim, N., N-phenylmaleimide polymers for second-order nonlinear optics, Polymer, 38, pp.4651-4656, 1997.

[12] Shen, N.X., Guo, T.Y., Yuan, X.Y. \& Sheng, J., Synthesis and Study for Styrene and N-phenyl Maleimide Copolymer, Polym. Mater Sci Eng, 13, pp.39-43, 1997.

[13] Dinakaran, K. \& Alagar M., Preparation and characterization of epoxy-cyanate ester interpenetrating network matrices/organoclay nanocomposites, Polymer for advanced technologies, 14, pp.574-585, 2003. 